\section{Krebs als Kind erhöht das Frühgeburtsrisiko}

\begin{abstract}
Bekommen Frauen, die als Kind an Krebs erkrankt waren, Kinder, werden diese oft zu früh und mit einem zu niedrigen Geburtsgewicht geboren. Dies zeigen verschiedene Untersuchungen. Unklar war bisher, ob Ähnliches auch auf Frauen mit Krebsdiagnose und -therapie im gebärfähigen Alter zutrifft.
\end{abstract}

G rundlage der Studie sind Daten des zentralen Krebs- und Geburtsregisters von North Carolina, USA. Die Daten von Frauen, die zwischen 2000 und 2013 im Alter von 15-39 Jahren an Krebs erkrankt waren, wurden mit den Lebendgeburten zwischen Januar 2000 und De-

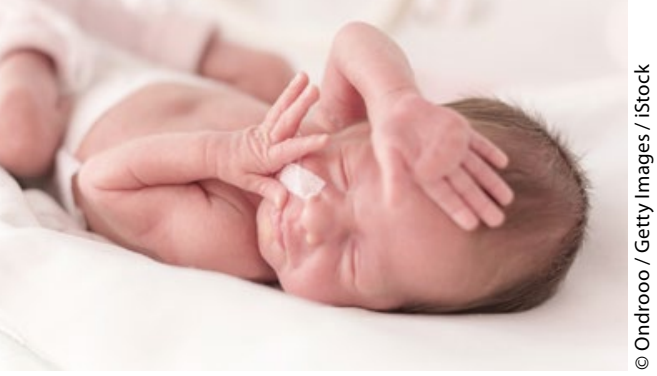

Zu früh oder zu leicht geboren: Das droht vor allem, wenn die Mutter Krebs hatte. zember 2014 abgeglichen. Von den 21.716 Frauen, bei denen im Studienzeitraum Krebs diagnostiziert worden war, brachten 8.529 insgesamt 14.132 Kinder lebend zur Welt. In der Vergleichsgruppe ohne Krebsdiagnose waren es knapp 13.000 Geburten. Berücksichtigt wurde jeweils nur die erste Geburt nach der Krebsdiagnose, und ein Geburtsgewicht unter $2.500 \mathrm{~g}$ wurde als niedrig eingestuft.

Für die Studie wurden letztlich die Daten von knapp 2.600 Geburten in der Gruppe der Krebspatientinnen analysiert. Die Frauen waren zum Zeitpunkt der Geburt durchschnittlich 31 Jahre alt. Vergleichsgruppe waren knapp 1.300 Geburten bei Frauen, die nicht an Krebs erkrankt waren. Häufige Krebsdiagnosen waren Melanom/Hautkrebs (21\%), Schilddrüsenkarzinom (19\%) und Brustkrebs (14\%). $85 \%$ der Patientinnen wurden operiert, $23 \%$ erhielten eine Bestrahlung und $25 \%$ eine Chemotherapie.
Das Risiko für eine Frühgeburt ist bei Krebspatientinnen im Vergleich zu gesunden Frauen signifikant höher. Die Prävalenz-Ratio (PR, der Quotient der Prävalenz bei Krebspatientinnen und Gesunden), lag in der Studie bei 1,52. Ähnlich hoch war das Risiko für ein geringes Geburtsgewicht (PR 1,59).

Die Wahrscheinlichkeit für eine Frühgeburt war bei Frauen mit gynäkologischen Tumoren besonders erhöht (PR $2,58)$, ebenso die für niedriges Geburtsgewicht (PR 2,74). Außerdem war das Risiko für eine Frühgeburt bzw. ein niedriges Geburtsgewicht vor allem nach einer Chemotherapie ohne Bestrahlung erhöht (PR 2,11 bzw. 2,36).

Fazit: Frauen mit Kinderwunsch, die im gebärfähigen Alter an Krebs erkranken und deshalb behandelt werden, müssen damit rechnen, dass ihr Kind zu früh auf die Welt kommt oder dass es ein niedriges Geburtsgewicht hat. Das erhöhte Risiko wurde in der Studie vor allem bei Frauen beobachtet, die chemotherapeutisch behandelt wurden. Peter Leiner

Anderson C et al. Birth Outcomes Among Adolescent and Young Adult Cancer Survivors. JAMA Oncol. 2017 Mar 23. [Epub ahead of print]

\section{Gebrechlichkeit bei Krebspatienten einschätzen}

Welche Krebstherapie ein älterer Patient erhält - und ob überhaupt noch eine möglich ist - sollte in erheblichem Maße von dessen Konstitution abhängen. Aber wie misst man Gebrechlichkeit am besten? Französische Forscher haben die gängigen Klassifikationen miteinander verglichen.

W ie gebrechlich ist ein Krebspatient? Es gibt viele „Frailty-Klassifikationen“, die bei Entscheidungen über Krebsund geriatrische Interventionen bei betagten Patienten helfen sollen, aber keinen Konsens darüber, wie man Gebrechlichkeit misst. Die Vorhersagekraft verschiedener Ansätze wurde nie wirklich evaluiert, auch nicht die der Empfehlungen der International Society of Geriatric Oncology zum geriatrischen Assessment (SIOG1 und SIOG2). Die Elderly Cancer Patients (ELCAPA) Study Group verglich 4 gebräuchliche Klassifikationen (SIOG1 und 2, die Klassifikation nach Balducci und die latente Klassentypologie [LC-Typologie]) bei einer Kohorte von 1.021 älteren Patienten ( $\geq 70$ Jahre). Die Patienten waren aufgrund verschiedener solider oder hämatologischer Malignome stationär oder ambulant behandelt worden. 763 Patienten wurden nach einer der 4 Klassifikationen beurteilt. Endpunkte waren die 1-Jahres-Mortalität und ungeplante Krankenhauseinweisungen binnen 6 Monaten.

Alle 4 Klassifikationen konnten die Patienten nach der 1-Jahres-Sterblichkeit gut unterteilen (C-Index $\geq 0,7$ ), am besten SIOG1. Auch bei ungeplanten
Klinikeinweisungen bewährten sich alle 4 Systeme (C-Index $\geq 0,7)$. Für die Klassifizierung in 3 (fit, vulnerabel oder gebrechlich) oder 2 Kategorien (fit vs. vulnerabel oder gebrechlich sowie fit oder vulnerabel vs. gebrechlich) lag die Übereinstimmung zwischen schlecht $(\kappa \leq 0,2)$ und gut $(0,6<\kappa \leq 0,8)$. Am besten stimmten SIOG1 und die LC-Typologie sowie SIOG1 und die Klassifikation nach Balducci überein.

Fazit: Die 4 Frailty-Klassifikationen haben insgesamt eine gute Vorhersagekraft für ältere stationäre und ambulante $\mathrm{Pa}$ tienten mit verschiedenen Krebsarten. Sie können bei der Auswahl von Krebstherapien und geriatrischen Interventionen nützlich sein.

Christina Berndt

Ferrat E et al. Performance of Four Frailty Classifications in Older Patients With Cancer: Prospective Elderly Cancer Patients Cohort Study. J Clin Oncol. 2017;35(7):766-77. 